\title{
新型绿色建筑工程造价预算及成本控制的分析
}

\author{
黄丽红 \\ 天颂建设集团有限公司杭州分公司，浙江 杭州 310000
}

[摘要] 随着自然资源的节约和生态环境保护的概念不断地在社会上兴起，中国新的绿色环保的建筑类型正在农村和城市地区 迅速普及、发展。绿色建筑的目标是实现人们生活和工作的大环境的和谐、美丽、舒适，让人真正的和自然平衡的共处。在 整个建筑工程项目的生命周期内，必须要深入的考虑到对能源的保护和高效率的使用。通过建筑物的墙体保温隔热材料，能 够减少建筑物的热交换，减少能源损耗。保证人们的健康、舒适、生态的居住空间，绿色环保建筑工程项目的新型设计应充 分利用自然资源、如阳光、地热等等, 为了更好的实现节约能源、保护环境的目标, 使居民的生活更接近生态环保、绿色节 约的理念。为了更加有效地提高绿色环保的建筑工程项目的总体质量和经济、生态效益, 对建筑工程项目的设计和控制工程 项目的成本的研究已成为这个领域的热点和重点。成本管理和控制工作是实现建筑工程项目的成本节约和提高建筑工程项目 经济效益和生态环保效益的关键。

[关键词]新型绿色建筑; 造价预算; 成本控制

DOI: $10.33142 /$ ec.v3i8.2361

中图分类号: TU723.3

文献标识码：A

\section{Analysis of Cost Budget and Cost Control of New Type Green Building Project}

\author{
HUANG Lihong
}

Tiansong Construction Group Co., Ltd. Hangzhou Branch, Hangzhou, Zhejiang, 310000, China

\begin{abstract}
With the concept of conservation of natural resources and ecological environment protection rising in society, new green building types in China are rapidly popularized and developed in rural and urban areas. The goal of green building is to realize the harmony, beauty and comfort of people's living and working environment, and let people live in balance with nature. In the life cycle of the whole construction project, we must consider the protection of energy and efficient use. Through the wall thermal insulation materials of buildings, the heat exchange of buildings can be reduced, and the energy consumption can be reduced. To ensure people's healthy, comfortable and ecological living space, the new design of green environmental protection building project should make full use of natural resources, such as sunlight, geothermal, etc., in order to better achieve the goal of energy saving and environmental protection, and make the residents' life closer to the concept of ecological environment protection and green saving. In order to effectively improve the overall quality, economic and ecological benefits of green and environmental protection construction projects, the research on the design and cost control of construction projects has become a hot spot and focus in this field. Cost management and control work is the key to realize the cost saving of construction projects and improve the economic and ecological benefits of construction projects.
\end{abstract}

Keywords: new green building; cost budget; cost control

\section{引言}

新型的绿色生态环保的建筑是充分的符合人们生存发展的可持续性要求的, 也顺应了人与自然和谐共生的根本要 求, 是建筑业未来发展的重要方向。新的生态环保的绿色建筑类型的成本控制方法与传统的建筑工程项目的成本控制 方法有很大的不同, 这就要求建筑企业的有关管理人员进行深入的研究、分析和探讨。

\section{1 新型绿色建筑的概念分析}

在当前生态环保的理念不断深入人心的时代下, 新的绿色环保的建筑工程项目建设的主要目标是更好的满足人民 生存发展的需要以及对美好的、生态的生活环境的需求。此外, 与传统的建筑工程项目的施工建设相比, 新的绿色环 保的建筑工程项目在施工建设方面包括许多新的设计施工的元素以及新的理念。绿色环保的建筑工程项目不仅包括整 个建筑工程项目施工建设的生态环境保护的要求, 而且还包括建筑工程项目的整体设计、景观建设和其他方面的环保 属性。可以清楚地看到, 绿色环保的建筑工程项目的维护管理是非常普遍的, 主要是为了更好的实现绿色环保的要求 和有效的控制整个建筑工程项目的环境 ${ }^{[1]}$ 。绿色建筑的施工建设的方法主要是在建筑工程项目施工建造的过程中合理使 
用一些环保、节能的建筑材料, 为了真正的实现节能和环保的绿色建筑的发展目标, 同时需要根据实际的工程项目情

况, 积极的优化项目建设的成本, 促进绿色环保的建筑工程行业的可持续发展。

\section{2 新型绿色建筑工程造价预算及成本控制模式}

建筑工程项目的施工建设的管理工作不仅要关注工程项目的建造质量和施工的安全, 同时也应侧重于工程项目的 施工预算和成本方面的管理控制, 这也可以进一步的保证建筑工程项目施工单位的经济效益的实现 ${ }^{[2]}$ 。

\section{1 工程预算的定额计价模式}

当前, 新型绿色建筑的理念被建筑行业普遍接受, 要想对新型绿色建筑进行造价预算及成本控制, 需要从其理念 入手，系统分析新型绿色建筑在建筑材料、项目资金、设备投入以及人工等方面的资金消耗，进行整体资金消耗的预 测, 制定出工程预算的最佳方案。

\section{2 工程量清单计价模式}

在工程招标时, 造价人员应根据绿色建筑工程的实际情况, 一方面要对施工单位的管理情况、财务情况以及技术 情况有着深入的了解; 另一方面还要求造价人员对招标模式有着清楚的认知, 谨防招标过程中出现暗箱操作等行为, 以确保新型绿色建筑工程的整体收益 ${ }^{[3]}$ 。

\section{3 绿色建筑成本管理原则}

\section{1 建筑生命周期设计原则}

建筑工程项目的整个生命周期, 指的是从建筑工程项目的最初的规划和设计, 一直到后续的施工建设以及竣工验 收和投入使用以及直到最后到建筑工程项目被拆除的整个环节。在建筑工程项目的整个生命周期当中, 都必须充分考 虑其成本费用。从建筑工程项目的经济效益和社会效益的角度来看, 绿色环保的建筑工程项目必须全面考虑和合理规 划工程项目在试用阶段的维修和管理的各类费用, 同时其成本管理和控制也必须从生态环境保护和节能的角度进行高 标准控制。

\section{2 资源综合高效利用原则}

在传统的建筑工程项目的施工建设的过程中, 各种成本费用的计算一般是按不同的类别、非常模糊的进行成本计 算的, 这给成本控制工作带来了很大的难题。所以说在绿色环保的建筑工程项目的成本管理工作中, 必须要充分的考 虑到所有影响工程项目成本的各种因素, 不断优化和完善整个建筑工程项目的设计, 选择那些生态的、绿色的、节能 的、无害环境的建筑材料, 同时高度合理的配置所有的建筑资源, 以不断提高建筑工程项目各类资源和材料的利用效 率, 真正的实现节能环保的总体目标, 可以在更高水平上上减少建筑施工企业的工程建造的成本 ${ }^{[4]}$ 。

\section{4 新型的绿色建筑工程造价预算分析}

管理一个新类型的绿色环保的建筑工程项目的成本预算是一个相对复杂和困难的任务, 因此对新型绿色环保的建 筑工程项目进行成本预算编制的工作人员的专业技术水平和综合素质以及有关经验都提出了非常严格的标准和要求, 必须不断教育和培训工程项目的成本预算的编制人员, 充分提高他们的能力。与传统的建筑工程项目相比, 绿色环保 的建筑工程项目在设计、建筑材料和建造施工技术工艺和方法方面都深刻的体现了生态环境保护的内涵, 因此, 绿色 环保的建筑工程项目的成本费用在预算方面和传统的建筑工程项目预算大不相同。这就要求绿色环保的建筑工程项目 预算的工作人员具备高度的专业技能和综合能力, 不仅要掌握有关绿色环保建筑工程项目的最新法律和条例, 而且在 建筑工程项目的技术、工艺和成本控制方面也有坚实的专业基础。

\section{5 新型绿色建筑工程成本控制对策}

\section{1 构建完善的成本控制系统}

在建筑工程项目的施工建造的管理工作中, 许多建筑工程项目的施工单位往往采用的纵向管理模式, 以加强工程 项目施工成本的管理和控制。但是这种纵向的成本管理控制模式往往有着更为复杂的管理体系, 在信息和数据的交换 或传输的过程中很有可能会出现某些失误, 造成了对工程项目的成本控制有一些负面的影响。因此在新兴的建筑工程 项目的成本控制当中, 应选择更科学合理的方式, 尽量减少中间环节, 并采用一种更为高效率的成本管理和控制模式, 这有助于进一步提高工程项目的成本管理的质量和水平, 确保建筑工程项目的成本控制和管理的各种信息数据的准确 性, 与此同时工程项目的成本控制还要求建立一个系统完善的监督责任制, 为有关部门和有关工作人员设定不同的成 本管理和控制的目标任务, 并且有效的加强对有关人员和部门的监督和考核评价。 


\section{2 确保工程质量以及做好成本控制}

首先, 建筑工程项目的施工建设阶段的成本管理和控制工作需要结合工程项目的实际情况, 有针对性的进行, 高 水准的进行工程项目成本预算的编制工作。建筑工程项目的施工企业必须根据现行的国家有关标准和规定制定全面的 工程项目成本管理和控制计划, 并根据企业自身以及工程项目自身的情况选择最佳的成本管控计划, 全面深度的评估 建筑工程项目的建设费用、机电设备的安装费用以及人力资源费用等等, 并准备一个系统、科学的成本管理和控制报 告。同时必须要严格的保证工程项目的建造施工的质量。建筑公司必须根据工程项目的成本预算, 进行详细的施工成 本的拆分, 按照严格成本预算的标准进行各个方面的投资, 并采用完善的、先进的、适合的建筑施工的技术方法进行 工程项目的投资建设, 以标准化的管理方式做好所有方面的建筑工程施工管理, 以更好的保证工程项目建造的质量和 安全。最后, 建筑公司必须特别注意控制工程项目建设阶段的各类费用, 根据工程项目的预算标准, 高水平的、严格 的控制工程项目成本费用支出，同时确保工程项目的总体建造水平。

\section{3 控制发包环节和做好合同管理}

在实施新的绿色环保的建筑工程项目的过程中，必须要充分的结合绿色环保建筑的实际情况，进行高水平的、良 好的合同管理和控制, 以最大限度地避免各种由于合同不规范而带来的损失。合同管理对于工程项目的经济效益的实 现是非常重要的。建筑工程公司应遵循合同管理的标准和要求, 做好工程项目施工成本费用管理的各类工作, 并保留 和建筑合同有关的各种材料信息。如果出现特殊的纠纷或者问题情况那么就可以根据合同当中的规定进行处理。

\section{4 融入先进的科学技术}

由于科学和技术的不断发展, 传统方法已不再适用于现代建筑环境。许多先进的工程技术不断发展, 可以有效地 提高工程项目的成本管理的效率和质量, 因此, 利用科学和技术至关重要。绿色建筑项目的成本估算和控制涉及许多 复杂的计算。完全依靠人工计算, 这不仅是一个巨大的工作量, 而且也是一个非常复杂的任务。因此, 在这种情况下, 现代技术可以提供更快和更准确的计算。

\section{6 结语}

作为建筑业未来发展的重要方向, 新的绿色环保的建筑满足了可持续的发展的根本需要, 对于建筑工程项目的预 算编制和成本控制应注意选择新的模式和方法, 在工程项目的建造司公的成本管理中必须充分考虑到施工的方方面面, 采用先进的技术手段和实施综合的成本预算设计，最大限度地减少工程项目成本资金的消耗并高水平的控制成本，使 施工单位能够获得更大的经济效益。

\section{[参考文献]}

[1]戚中华. 新型绿色建筑工程造价预算与成本控制分析 [J].住宅与房地产, 2020(21): 29.

[2]张丽,张涛. 新型绿色建筑工程造价预算与成本控制分析 [J].居舍, 2020(20): 171-172.

[3] 言婷. 新型绿色建筑工程造价预算与成本控制分析 [J].农家参谋, 2020(12) : 228.

[4]宋仕忠,吕学良, 尚艳蕊. 新型绿色建筑工程造价预算与成本控制分析 [J].住宅与房地产, 2020(12): 25.

[5] 郭莉虹. 新型绿色建筑工程造价预算与成本控制分析 [J].四川水泥, 2020(03): 275.

作者简介: 黄丽红 (1982.6-), 女, 浙江大学, 土木工程, 天颂建设集团有限公司杭州分公司, 造价部经理, 助理工 程师。 